\section{Hydrocephalus, a rare manifestation of sarcoidosis}

\author{
Johan M. van Rooijen, ${ }^{1}$ \\ Gerritje S. Mijnhout, ${ }^{1}$ Tom T.A. Aalders, \\ R.B.J. de Bondt ${ }^{3}$ \\ Departments of ${ }^{1}$ Internal Medicine; \\ ${ }^{2}$ Neurosurgery; ${ }^{3}$ Radiology, Isala Clinics, \\ Zwolle, the Netherlands
}

\begin{abstract}
A 36-week-pregnant woman developed a symptomatic hydrocephalus. Chest imaging showed bihilar lymphadenopathy and histological examination of a mediastinal lymph node revealed non-caseating granulomas. After delivery, her neurologic complaints progressed. Placement of a ventriculoperitoneal drain (VPD) did not reduce the symptoms. However, steroids resulted in rapid disappearance of the hydrocephalus. Hydrocephalus is a very rare manifestation of sarcoidosis. The diagnosis relies on the ability of clinicians to recognize this disorder. This case shows how a difference in opinion of the several specialists involved can lead to a delay in diagnosis and treatment.
\end{abstract}

\section{Introduction}

Sarcoidosis is a multisystem granulomatous disorder of unknown origin typically affecting young adults. The organs most commonly involved are the lungs, skin and lymph nodes. Involvement of the nervous system occurs in $5 \%$ of patients with sarcoidosis. ${ }^{1}$ Neurological involvement increases morbidity and mortality significantly. ${ }^{2}$ Seventh cranial nerve involvement with unilateral facial palsy is the most common finding. ${ }^{3}$ Neuritis of the optical nerve is the second most common cranial nerve neuropathy. Other presentations of neurosarcoidosis include aseptic meningitis, hearing abnormalities, headache, vertigo, pituitary dysfunction, cerebral mass lesions, myopathy, seizures and psychiatric symptoms. ${ }^{3}$ Hydrocephalus, however, is a very uncommon presenting symptom of sarcoidosis. ${ }^{3,4,5}$

Of all manifestations of neurosarcoidosis, hydrocephalus has the worst long-term prognosis with a mortality rate of $75 \% .^{3,4}$ Occasionally, neurosarcoidosis can cause unexpected sudden death. ${ }^{6}$

We present a patient with a hydrocephalus as a presenting manifestation of sarcoidosis. To our knowledge only few cases have been reported. $3,4,5,7$

\section{Case Report}

A 30-year-old woman, 36-week-pregnant of her second child, was admitted to the department of gynecology for a symptomatic hydrocephalus. She had a 6 -week history of progressive nausea, vomiting, dizziness, diplopia and headache precipitated by position change. Cranial magnetic resonance imaging (MRI) showed hydrocephalus with enlargement of the entire ventricle system, including the fourth ventricle, and transependymal effusion of cerebrospinal fluid (CSF), see Figure 1A and 2A. No cerebral mass lesions and no evident obstruction could be demonstrated, suggesting a communicating hydrocephalus due to decreased resorption of CSF. However, the signal intensities of the CSF in the posterior fossa (Figure 3) could suggest a membranous outflow-obstruction at the foramina of Luschka. Physical examination was normal except for mild cognitive dysfunction, nystagmus and disturbed coordination. Papilledema was not present. There was no neurological evidence of a cranial nerve palsy. The internist was asked to evaluate for possible underlying internal causes of a communicating hydrocephalus. A detailed medical history revealed that during the past 6 years, she had had three episodes of an anterior uveitis of unknown origin. In addition, a few years ago she had had possible arthritis of her ankles, wrists and elbows, responding well to a non-steroidal anti-inflammatory drug. The course of her first pregnancy had been unremarkable. Laboratory tests only showed a slightly increased ESR. Anti-nuclear antibodies, anti-dsDNA and ANCA's were negative. A proposed diagnostic lumbar puncture was found to be an absolute contra-indication by the neurosurgeon because of the risk of downward herniation. Chest X-ray showed bihilar lymphadenopathy. An additional computed tomography (CT)-scan of the chest, performed without intravenous contrast and with a limited number of slices to minimize the amount of radiation, showed a reticulonodular pattern and bilateral hilar lymphadenopathy suggestive of sarcoidosis. Serum ACE was not raised, but 1.25-dihydroxyvitamin $\mathrm{D}$ was increased (175 pmol/L). Due to the pregnancy, endobronchial ultrasound with a fine needle aspiration (FNA) of enlarged lymph nodes was not performed. Because of the bilateral hilar lymphadenopathy on chest imaging and the history of uveitis and arthritis, neurosarcoidosis was considered the most likely cause of the hydrocephalus. The internist advised immediate treatment with high dose prednisolone, to reduce the hydrocephalus and the chance of neurological complications due to increased intracranial pressure during a vaginal labor. This advice however was not followed, because of a difference in opinion of the several spe-
Correspondence: Gerritje S. Mijnhout, Department of Internal Medicine, Isala Clinics, P.O. Box 10400, 8000 GK Zwolle, the Netherlands.

Tel. +38.4247713 - Fax: +38.4243367.

E-mail: g.s.mijnhout@isala.nl

Key words: sarcoidosis, hydrocephalus, neurosarcoidosis.

Received for publication: 14 July 2011.

Accepted for publication: 22 August 2011.

This work is licensed under a Creative Commons Attribution NonCommercial 3.0 License (CC BYNC 3.0).

(C) Copyright J.M. van Rooijen et al., 2011

Licensee PAGEPress, Italy

Clinics and Practice 2011; 1:e66

doi:10.4081/cp.2011.e66

cialists involved. Because the patient remained neurologically stable, placement of a VPD was not performed. At 37-weeks of gestation the patient delivered a healthy daughter after cervical priming. Initially, neurologic complaints reduced. However, one week later she developed intolerable headache, nausea and vomiting. In addition, her vision was subjectively blurred without evidence of papilledema. A VPD was placed immediately. CSF pressure was elevated with an opening pressure of $28 \mathrm{~cm} \mathrm{H2O}$. Analysis of the CSF showed a low glucose at $2.2 \mathrm{mmol} / \mathrm{L}$, increased total protein at $1.32 \mathrm{~g} / \mathrm{L}$ with increased albumin quotient, a lymphocytic pleiocytosis and an elevated IgG index, compatible with chronic inflammation. CSF culture was negative. Borrelia serology of the liquor was negative and no tumour cells were found. CSF ACE level was not elevated $(<1 \mathrm{U} / \mathrm{L})$. The shunt failed to reduce the symptoms. Cranial CT scan showed no change in dilatation of the ventricular system. Re-exploration of the VPD was performed but showed no signs of shunt dysfunction. Therefore, the internist was consulted again. FNA cytology of an enlarged mediastinal lymph node was performed, followed by treatment with prednisolone $(1 \mathrm{mg} / \mathrm{kg})$ on suspicion of neurosarcoidosis. Histological examination of a mediastinal lymph node showed non-caseating granulomas, compatible with sarcoidosis. Within 3 weeks after the start of prednisolone, the patient became asymptomatic and repeated cranial MRI showed complete resolution of the hydrocephalus and transependymal effusion of CSF (Figure 1B and 2B).

\section{Discussion}

Hydrocephalus is a rare manifestation of 

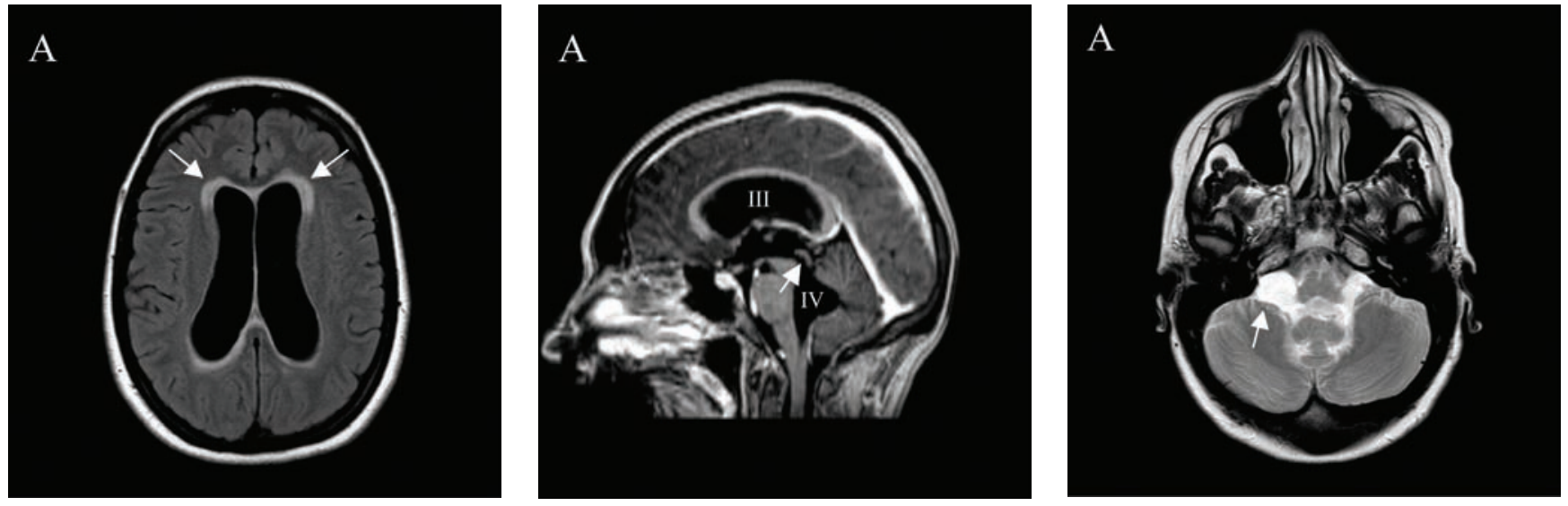

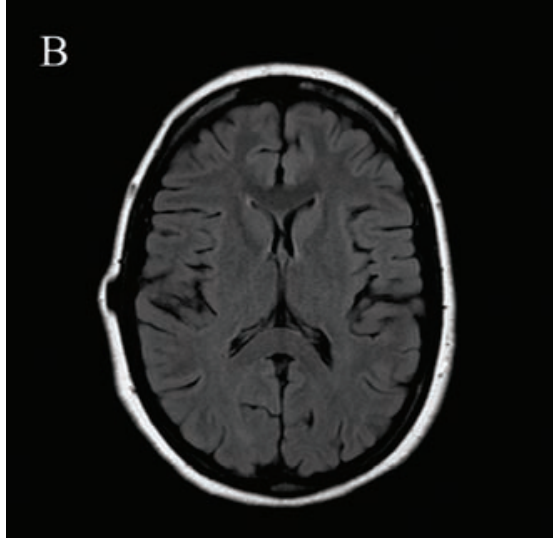

Figure 1. Magnetic resonance imaging of the head, axial FLAIR images at the level of the lateral ventricles. (A) First scan at presentation, showing a dilated ventricular system accompanied with periventricular transependymal effusion of CSF (arrows). (B) Placement of a VPD did not change the hydrocephalus, but after three weeks of therapy with steroids normalization of the ventricles width and resolution of the transependymal effusion of CSF is demonstrated.

sarcoidosis. Sarcoidosis presenting with a hydrocephalus is even rarer and poses a substantial diagnostic dilemma. ${ }^{3,5}$ Adequate diagnosis relies upon the ability of clinicians to recognize this disorder. This case shows that the internist can play an important role in the diagnosis of hydrocephalus. Many internal diseases can cause a hydrocephalus. Particularly infections, tumours and autoimmune disorders have to be considered. ${ }^{8}$ (Table 1 )

Sarcoidosis can cause an obstructive hydrocephalus by fourth ventricular outflow obstruction. ${ }^{4}$ In this case, the MRI at presentation at the level of the posterior fossa suggested a membranous outflow-obstruction at the foramina of Luschka, without showing any obstruc-

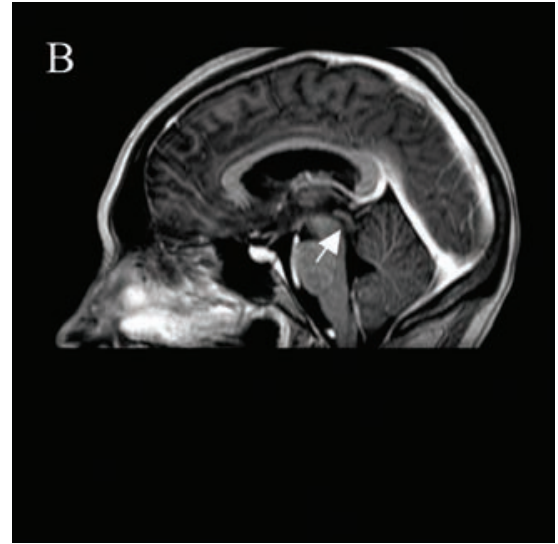

Figure 2. Magnetic resonance imaging of the head, median sagittal T1 weighted images post contrast injection. (A) Dilated third en fourth ventricles and dilated aquaduct (arrow) can be appreciated. There are no signs of pathological ependymal enhancement or enhancing masses.(B) Placement of a VPD did not change the hydrocephalus, but after three weeks of therapy with steroids, normal size and aspect of the third and fourth ventricle and aquaduct (arrow) is observed.

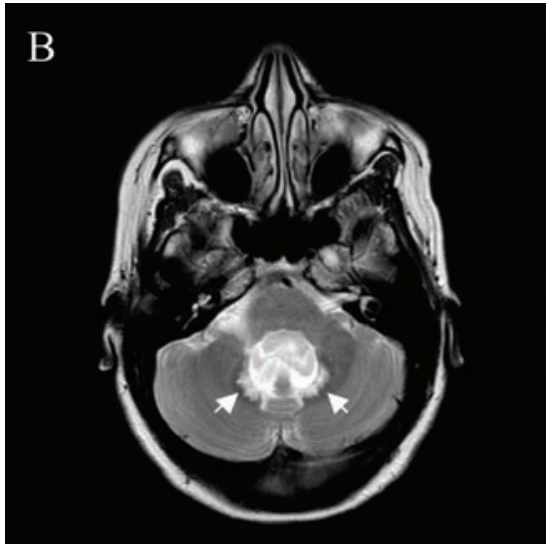

Figure 3. Magnetic resonance imaging of the head, axial T2 weighted images at the level of posterior fossa. First scan at presentation, showing (A) enlargement of the cerebellomedullary cisterns. The signal intensities of the CSF (arrow) suggest a membranous outflow-obstruction at the foramina of Luschka, although no cerebral mass lesions and no obstruction could be demonstrated. (B) This image shows an enlarged fourth ventricle and a significant transependymal effusion of CSF dorsally (arrows), a phenomenon not seen very often in the posterior fossa.

Table 1. Internal causes of an acquired hydrocephalus in adults.

Benign and malignant tumors (including leptomeningeal metastases)

Meningitis/Encephalitis (bacterial, fungal, viral, including EBV, HIV and syphilis)

Lyme disease (neuroborreliosis)

Toxoplasmosis

Tuberculosis

Sarcoidosis

Systemic lupus erythematodus (SLE)

Wegener's granulomatosis

Behçet's disease

Whipple's disease

Neurocysticercosis 
tion due to mass lesions. However, conventional T1- and T2-weighted MRI images are insensitive to obstructive membranes in CSF pathways, especially in the fourth ventricular exit foramina and the basal cisterns. ${ }^{9}$ This may lead to misclassification of a non-communicating hydrocephalus as a communicating hydrocephalus.

Sarcoidosis can also lead to a reduced absorption of CSF in the basal cisterns, resulting in a communicating hydrocephalus, ${ }^{4}$ probably due to inflammatory induced dysfunction of the arachnoidal villi. This is presumably the case in our patient. We cannot explain why the hydrocephalus did not disappear after placement of the VPD. In the literature, shunt failure has been reported due to occlusion with non-caseating granulomatous material that had infiltrated the shunt lumen, ${ }^{10}$ but in our case shunt dysfunction was excluded.

The diagnosis of neurosarcoidosis is based on exclusion of other possible causes and searching for extra-neurologic manifestations, preferably with pathological analysis of suspected tissue (e.g. enlarged lymph nodes) to demonstrate non-caseating granulomas. The diagnosis is often delayed, potentially leading to serious complications. ${ }^{11}$

Diagnostic criteria for neurosarcoidosis have been proposed by Zajicek et al. ${ }^{8}$ who define possible, probable and definite neurosarcoidosis (Table 2). ${ }^{8}$ A nervous system biopsy is necessary to establish a diagnosis of "definite" neurosarcoidosis, however this is not always feasible. Our patient met the criteria of "probable" neurosarcoidosis. We did not perform a nervous system biopsy because of the absence of cerebral mass lesions.

CSF ACE level is frequently normal and this test is not sensitive enough to exclude neurosarcoidosis. $^{12}$

There is no consensus on the optimal treatment of patients with neurosarcoidosis, as clinical trials are not available. However highdose corticosteroids (prednisolone $1 \mathrm{mg} / \mathrm{kg}$ ) are widely accepted as the first line of treatment. ${ }^{13}$ Patients usually respond within days to weeks. $^{14}$ Alternative immunosuppressive agents, including methotrexate, azathioprine, cyclosporine, infliximab and cyclofosfamide can be used in case of failure or intolerance to steroids. ${ }^{14}$ If steroids had been started earlier in our patient, placement of a VPD might not have been necessary. ${ }^{3}$

Table 2. Diagnostic criteria for neurosarcoidosis (modified from Zajicek et al.).

Neurosarcoidosis (NS) can be diagnosed in patients with a clinical presentation suggestive of neurosarcoidosis with exclusion of other possible diagnoses, as follows:

1. Definite NS: Positive central nervous system histology.

2. Probable NS: (a) Laboratory evidence of central nervous system inflammation (elevated levels of CSF protein and/or cells, the presence of oligoclonal bands and/or MRI evidence compatible with neurosarcoidosis), and (b) Evidence for systemic sarcoidosis (either through positive histology and/or at least two indirect indicators from Gallium scan, chest imaging and serum ACE).

3. Possible NS: when the above criteria are not met.

\section{Conclusions}

Hydrocephalus is a rare manifestation of sarcoidosis. This case shows that diagnosing neurosarcoidosis relies on the ability of clinicians to recognize this disorder; difference in opinion of the involved specialists can lead to a delay in diagnosis and treatment. The diagnosis can be difficult when hydrocephalus is the presenting symptom of sarcoidosis.

A systemic evaluation of all possible neurologic and extra-neurologic manifestations must be performed. Because an acquired hydrocephalus in adults can have many non-neurological causes, we advocate a multidisciplinary approach to diagnosis and treatment. Treatment of hydrocephalus due to neurosarcoidosis consists of high dose steroids and on demand placement of a VPD. However, placement of a VPD alone, without steroid treatment, will not cure the patient from neurosarcoidosis.

\section{References}

1. Spencer TS, Campellone JV, Maldonado I, et al. Clinical and magnetic resonance imaging manifestations of neurosarcoidosis. Semin Arthritis Rheum 2004;34:649-61.

2. Lower EE, Broderick JP, Brott TG, et al. Diagnosis and management of neurological sarcoidosis. Arch Intern Med 1997;157: 1864-8.

3. Akhondi H, Barochia S, Holmström B, Williams MJ. Hydrocephalus as a presenting manifestation of neurosarcoidosis. Southern Med J 2003;96:403-6.

4. Benzagmout M, Boujraf S, Góngora-Rivera F, et al. Neurosarcoidosis which manifested as acute hydrocephalus: diagnosis and treatment. Intern Med 2007;46:1601-4.

5. Lee CH, Jung YS, Lee SH. Hydrocephalus as a presenting manifestation of neurosarcoidosis: easy to misdiagnose as tuberculosis. J Korean Neurosurg Soc 2010;48:7981.

6. Byard RW, Manton N, Tsokos M. Sarcoidosis and mechanisms of unexpected death. J Forensic Sci 2008;53:460-4.

7. Brouwer MC, de Gans J, Willemse RB, van de Beek D. Sarcoidosis presenting with hydrocephalus. J Neurol Neurosurg Psychiatry 2009;80:550-1.

8. Zajicek JP, Scolding NJ, Foster 0, et al. Central nervous system sarcoidosis - diagnosis and management. QJM 1999;92:103-17.

9. Dinçer A, Kohan S, Özek MM. Is all "communicating" hydrocephalus really communicating? Prospective study on the value of 3D-constructive interference in steady state sequence at 3T. AJNR Am J Neuroradiol 2009;30:1898-906.

10. Maniker AH, Cho ES, Schulder M. Neurosarcoid infiltration of the ventricular catheter causing shunt failure: a case report. Surg Neurol 1997;48:527-9.

11. Terushkin V, Stern BJ, Judson MA, et al. Neurosarcoidosis: presentations and management. Neurologist 2010;16:2-15.

12. Dale JC, O'Brien JF. Determination of angiotensin-converting enzyme levels in cerebrospinal fluid is not a useful test for the diagnosis of neurosarcoidosis. Mayo Clin Proc 1999;74:535.

13. Pawate S, Moses H, Sriram S. Presentations and outcomes of neurosarcoidosis: a study of 54 cases. QJM 2009;102:449-60.

14. Patel AV, Stickler DE, Tyor WR. Neurosarcoidosis. Curr Treat Options Neurol 2007;9:161-8. 